\title{
Structural characterization of ambazone salt with niflumic acid
}

\author{
Irina Kacso ${ }^{\text {a }}$, Lucia Rus ${ }^{\mathrm{b}}$, Mircea Pop ${ }^{\mathrm{a}}$, Gheorghe Borodi ${ }^{\mathrm{a}}$ and Ioan Bratu ${ }^{\mathrm{a}, *}$ \\ ${ }^{a}$ National Institute for Research and Development of Isotopic and Molecular Technologies, \\ Cluj-Napoca, Romania \\ ${ }^{\mathrm{b}}$ Department of Drug Analysis, Faculty of Pharmacy, University of Medicine and Pharmacy, \\ Cluj-Napoca, Romania
}

\begin{abstract}
Salt formation is a good method of increasing solubility, dissolution rate and consequently the bioavailability of poor soluble acidic or basic drugs [Polymorphism in Pharmaceutical Solids, Drugs and the Pharmaceutical Sciences, Vol. 192, 2nd edn, Informa Healthcare, New York, 2009]. The aim of this study was to obtain and to investigate the structural properties of the compound obtained by solvent drop grinding (SDG) method [Chem. Commun. (Camb.) 20 (2002), 2372-2373] at room temperature starting from the 1:1 molar ratios of ambazone (AMB) and niflumic acid (NIA). The thermal behavior of the obtained compound (AMB - NIA) was investigated by differential scanning calorimetry (DSC) and thermogravimetry (TG), the thermal diffusivity and effusivity parameters were determined by photothermal radiometry (PTR). The structural characterization was performed with X-ray powder diffraction (XRPD) and infrared spectroscopy (FTIR). XRPD data and FTIR spectra demonstrated a new structure for AMB · NIA compound as compared to those of the starting materials.
\end{abstract}

Keywords: Ambazone, solid forms, X-ray diffraction, FTIR spectroscopy, thermal analysis

\section{Introduction}

The most active areas of modern solid state chemistry represent the identification and characterization of different crystal forms (polymorphs, solvates, salts and co-crystals) of the same molecule. The investigation of crystal forms impacts on fundamental science because different crystal forms may display a range of different physical-chemical properties, which may affect application and utilization of the solid materials. Traditionally, the solid form selection process was limited to the free drug or pharmaceutically accepted salts. The solid form influences relevant physical-chemical parameters such as solubility, dissolution rate, chemical stability, melting point and hygroscopic parameter which can result in solids with superior properties [5,23]. In recent years, much of the research has been carried out on the preparation of pharmaceutical solid forms. Usually, there are two methods by which solid forms may be prepared: solution-based crystallization and grinding. Mechanical chemical methods $[8,9,17,20,22]$, more commonly and usefully described as grinding, have been employed extensively in the preparation of solid forms. The range of grinding conditions has been extended by the addition of solvents in the "solventdrop" method [5] and this may represents the introduction of solution conditions on a limited scale to the grinding process. More recently, the use of so-called "solvent-drop grinding" has been developed, in which a small quantity of a solvent is added to the solid substance or mixture prior to grinding.

\footnotetext{
*Corresponding author: Ioan Bratu, National Institute for Research and Development of Isotopic and Molecular Technologies, 65-103 Donáth street, 400293 Cluj-Napoca, Romania. E-mail: ibratu@gmail.com.
} 

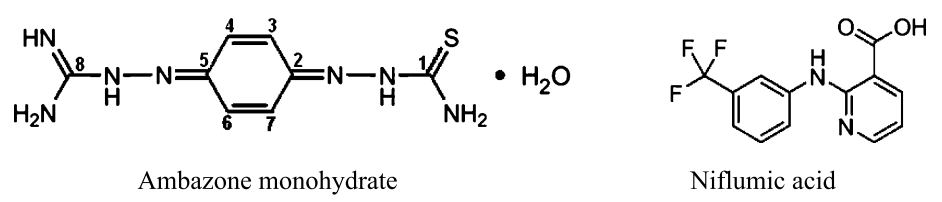

Fig. 1. Chemical structures of the starting materials.

Ambazone monohydrate, ([4-(2(diaminomethylidene)hydrazinyl)phenyl] iminothiourea), $\mathrm{C}_{8} \mathrm{H}_{11} \mathrm{~N}_{7} \mathrm{~S}$. $\mathrm{H}_{2} \mathrm{O}$ (AMB, see Fig. 1) is one of the oldest antimicrobial chemicals. The studies performed during the 1950-1960 decade have shown the local antibacterial properties when it is administrated at the buccal pharyngeal cavity level, being an efficient antimicrobial drug [11].

The ulterior re-evaluation of the antibacterial AMB properties evidenced an antibacterial activity spectrum similar to that of sulfamides [18]. Recently, the antineoplasm properties of AMB were also demonstrated $[1,2,4,10,12,15,16]$, that accelerated the researches on this substance, without mutagenic effects and unpleasant reactions characteristic to other oncostatic drugs [18].

Niflumic acid, (2-\{[3-(trifluoromethyl)phenyl]amino\}nicotinic acid), $\mathrm{C}_{13} \mathrm{H}_{9} \mathrm{~F}_{3} \mathrm{~N}_{2} \mathrm{O}_{2}$ (NIA, see Fig. 1), an analgesic and nonsteroidal anti-inflammatory drug [25] that also blocks or modifies the gating of many ion channels, is used in the treatment of rheumatoid arthritis [6].

The aim of this study was to obtain a salt of ambazone with niflumic acid (AMB - NIA) and to characterize its physical-chemical and structural properties using several investigation methods such as: X-ray diffraction, FTIR spectroscopy and several thermal methods (differential scanning calorimetry, thermogravimetry and photothermal radiometry).

\section{Experimental procedure}

\subsection{Materials and preparation}

AMB was obtained from Microsin SRL Bucharest, Romania, NIA commercially available was obtained from Alfa Aesar, Germany and these compounds were used without further purification. Solventdrop grinding (SDG) experiments were performed by placing $255.3 \mathrm{mg}$ AMB with 282.22 mg NIA (1:1 molar ratio) and grinding this mixture in an agate mortar by adding in drops $2 \mathrm{ml}$ ethanol at room temperature, until a dried compound was obtained. The resulting sample was analyzed using X-ray powder diffraction (XRPD), FTIR spectroscopic technique and thermal analysis methods (DSC, TG, PTR).

\subsection{X-ray powder diffraction}

XRPD patterns were obtained by a Bruker D8 Advance diffractometer, the measurements were performed at $20^{\circ} \mathrm{C}$ and were controlled by computer. The experimental conditions were: the $2 \theta$ range between 2.5 and $45^{\circ}, \mathrm{CuK} \alpha 1$ radiation $(\lambda=1.5406 \AA),(40 \mathrm{kV} ; 40 \mathrm{~mA}), \mathrm{Ge} 111$ monochromator on the diffracted beam. The step scan mode was performed with a step width of 0.01 at a rate of $1 \mathrm{step} / \mathrm{s}$. The samples were mildly pre-ground with a pestle in an agate mortar to make them homogeneous, to control crystals size and to minimize the preferred orientation effects. 


\subsection{FTIR spectroscopy}

The FTIR spectra were recorded with a JASCO 6100 FTIR spectrometer (number of scans, 256; resolution, $4 \mathrm{~cm}^{-1}$; range, $4000-400 \mathrm{~cm}^{-1}$ ). The $\mathrm{KBr}$ pellets were prepared by mixing $0.8 \mathrm{mg}$ of sample and $150 \mathrm{mg} \mathrm{KBr}$ and pressing the mixture into a $13 \mathrm{~mm}$ disks at 12 tones pressure. The spectra were analyzed using Spectra Analysis and Origin software.

\subsection{Thermal analysis DSC-TG}

Differential scanning calorimetry (DSC) was carried out by means of a Shimadzu DSC-60 calorimeter, the sample was heated in the range of $30-350^{\circ} \mathrm{C}$ with a heating rate of $10^{\circ} \mathrm{C} / \mathrm{min}$ in crimped aluminum sample cell. The purge gas was nitrogen with $60 \mathrm{ml} / \mathrm{min}$ flow. For data collection and analysis the Shimadzu TA-WS60 and TA60 2.1 software were employed.

TG curves were obtained with a TGA/SDTA 851e thermobalance. The samples were weighed (Mettler Toledo balance) directly in alumina pans (approximately $5 \mathrm{mg}$ ), and scanned between 25 and $400^{\circ} \mathrm{C}$ with a heating rate of $10^{\circ} \mathrm{C} / \mathrm{min}$ under dynamic nitrogen atmosphere $(50 \mathrm{ml} / \mathrm{min})$.

\subsection{Photothermal radiometry}

PTR technique has been used to measure the dynamic thermal parameters (thermal diffusivity and effusivity) of the pressed powder samples. The PTR technique consists in irradiating the sample with an optical radiation (YAG Laser) and measurement (with a $\mathrm{HgCdTe}$ sensor) of the emitted IR radiation.

\section{Results and discussion}

\subsection{X-ray powder diffraction}

X-ray powder patterns for AMB, NIA and AMB - NIA are shown in the Fig. 2.

From powder pattern indexing by using Dicvol method [19] it was established that AMB $\cdot$ NIA crystallizes in monoclinic system having following lattice parameters: $a=14.61 \AA, b=5.07 \AA, c=38.22 \AA$ and $\beta=111.62^{\circ}$.

The X-ray powder diffraction patterns of the AMB, NIA and AMB with NIA are clearly different, from these one can be see that the powder diffraction pattern of AMB - NIA solid form presents different features comparing with both of starting materials, that means a solid form of AMB with NIA was obtained an ambazone niflumate salt.

\subsection{FTIR spectroscopy}

The band at $\sim 3404 \mathrm{~cm}^{-1}$ can be assigned to $\mathrm{N}-\mathrm{H}$ stretching from primary amine in pure ambazone (see Fig. 3); the FTIR spectrum contains also [13,14] NH stretching band in the $3320-3180 \mathrm{~cm}^{-1}$ spectral range, i.e., $3226 \mathrm{~cm}^{-1}[13,24]$. The new band which appears at $\sim 3513 \mathrm{~cm}^{-1}$ in the spectrum of AMB - NIA can be explained by the hydrogen bond breaking between $\mathrm{H}_{2} \mathrm{O}$ molecule and $\mathrm{N}-\mathrm{H}$ group of AMB after the salt formation. Also it has been shown a change in the $\mathrm{NH}$ stretching absorption of amines [13]; it was observed that the sharp strong band at $\sim 3226 \mathrm{~cm}^{-1}$ due to the NH stretching is shifted to $3254 \mathrm{~cm}^{-1}$ and reduced in intensity. The band at $3147 \mathrm{~cm}^{-1}$ corresponds to the $\mathrm{NH}$ vibration 


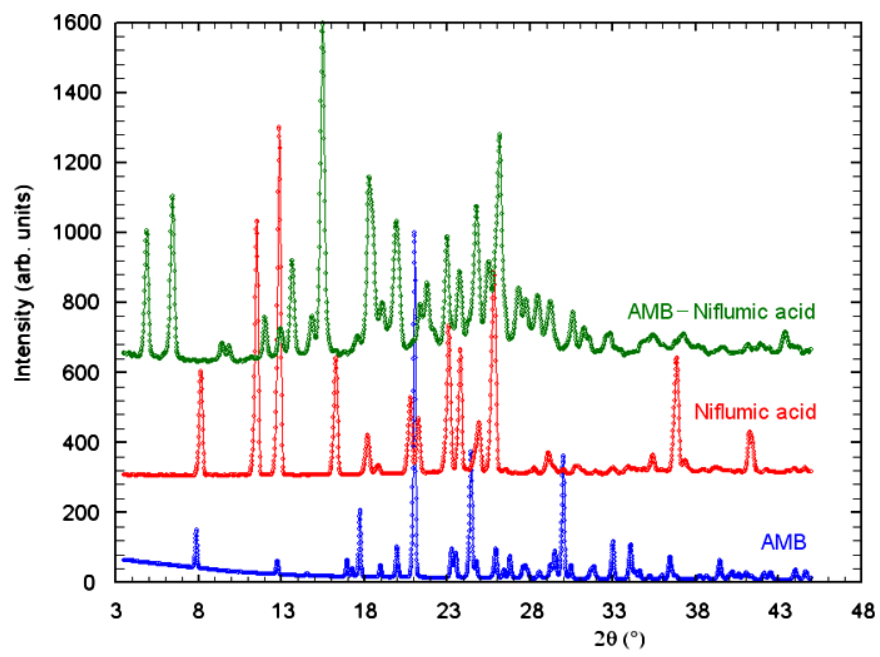

Fig. 2. X-ray powder diffraction patterns for AMB, NIA and AMB - NIA obtained by SDG. (Colors are visible in the online version of the article; http://dx.doi.org/10.3233/SPE-2012-0570.)

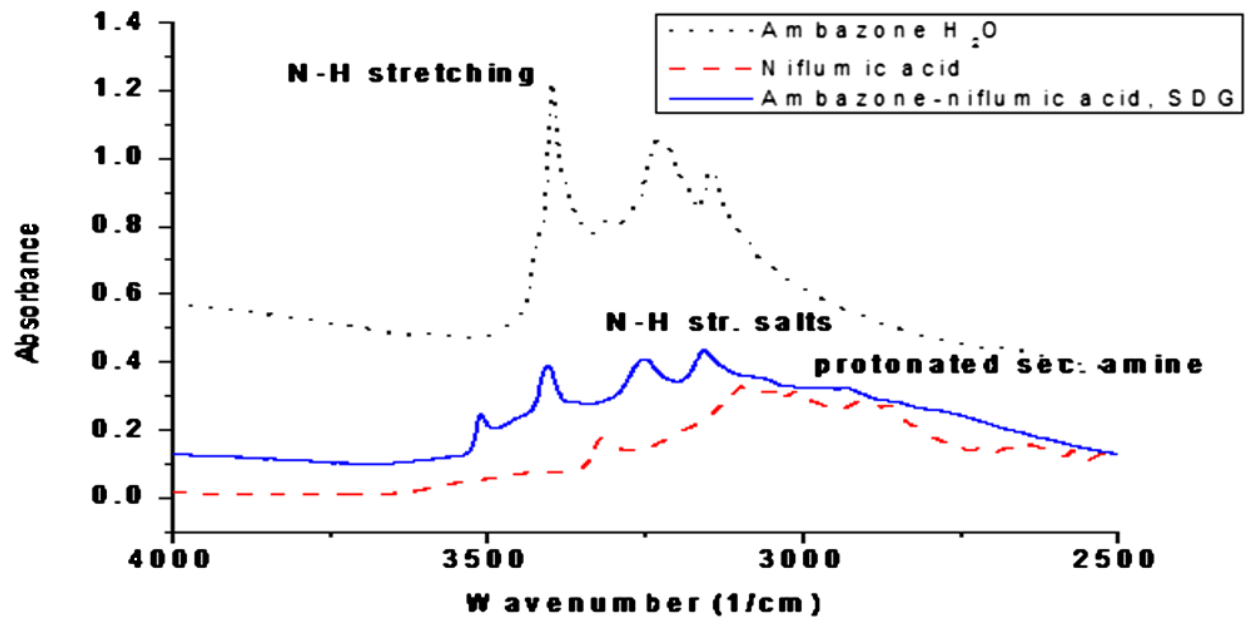

Fig. 3. FTIR spectra of AMB, NIA and their salt obtained by SDG method, 4000-2500 $\mathrm{cm}^{-1}$ spectral range. (Colors are visible in the online version of the article; http://dx.doi.org/10.3233/SPE-2012-0570.)

[13] for pure AMB, this band shifted at $3158 \mathrm{~cm}^{-1}$ in the salt spectrum. A new shoulder appeared at $\sim 2937 \mathrm{~cm}^{-1}$ probably due to the protonated secondary amine.

The spectrum of the pure NIA contains the NH stretching vibration at $3321 \mathrm{~cm}^{-1}$ and a broad signal at $3090 \mathrm{~cm}^{-1}$ assigned to stretching vibration of $\mathrm{CH}$ groups from benzene ring [3]. These signals do not appear in the spectrum of the AMB - NIA.

Primary amine has an absorption band of medium intensity at $\sim 1613 \mathrm{~cm}^{-1}$ (see Fig. 4), being shifted to $\sim 1601 \mathrm{~cm}^{-1}$ by salt formation [13]. Cleaves and Plyler [7] correlated the spectral bands at 1625$1516 \mathrm{~cm}^{-1}$ with $\mathrm{NH}$ deformation vibration. The pure ambazone spectrum contains the secondary amine vibration at $1508 \mathrm{~cm}^{-1}$ which is shifted in AMB . NIA spectrum to $1516 \mathrm{~cm}^{-1}$ and greatly reduced its intensity. 


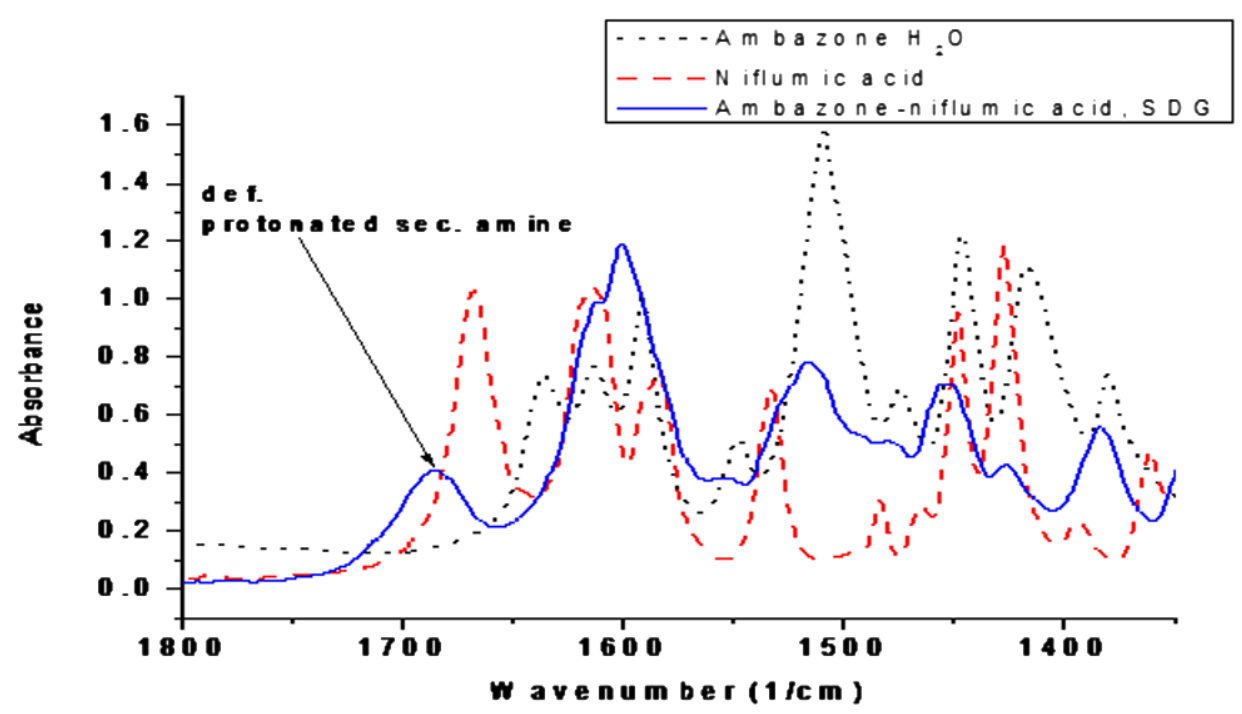

Fig. 4. FTIR spectra of AMB, NIA and their salt obtained by SDG method, $1800-1000 \mathrm{~cm}^{-1}$ spectral range. (Colors are visible in the online version of the article; http://dx.doi.org/10.3233/SPE-2012-0570.)

In the spectrum of the pure NIA a strong absorption band appears at the $1668 \mathrm{~cm}^{-1}$ corresponding to the carboxylic CO stretching vibration [3]; this band shifted in the AMB - NIA spectrum to $1686 \mathrm{~cm}^{-1}$, due to the deprotonation of the carboxyl group for aryl compounds [19] (Fig. 4). This absorption located at $\sim 1686 \mathrm{~cm}^{-1}$, can be assigned also to deformation vibration of the protonated secondary amino group [7]. The benzene ring stretching vibration at $1614 \mathrm{~cm}^{-1}$ is not shifted and appeared as a shoulder on the spectrum of the AMB - NIA. At $1428 \mathrm{~cm}^{-1}$ an intense spectral band attributed to carboxylic $\mathrm{C}=\mathrm{O}$ and $\mathrm{C}-\mathrm{C}$ stretching and $\mathrm{OH}$ rocking vibrations appears, it decreases in intensity, probably due to the carboxyl involving in intermolecular interactions. The shift of the vibrational mode at $\sim 1448-1454 \mathrm{~cm}^{-1}$ can be due to the influence of the deprotonated carboxyl group on the pyridine ring.

\subsection{Thermal analysis $D S C-T G$}

The DSC thermograms of the pure AMB, NIA and of the AMB $\cdot$ NIA compound obtained by SDG are presented in Fig. 5. The curve for the pure AMB revealed a broad endothermic signal from 105 to $143^{\circ} \mathrm{C}$, with a maximum at $125^{\circ} \mathrm{C}$ and $\Delta H=36 \mathrm{~kJ} / \mathrm{mol}$, that corresponds to the water molecules loss of the AMB monohydrate structure, followed by a sharp exothermic signal at $204.52^{\circ} \mathrm{C}, \Delta H=75 \mathrm{~kJ} / \mathrm{mol}$ due to the melting with decomposition of AMB.

The thermogram of the pure NIA revealed a sharp endothermic signal with maximum at $204.75^{\circ} \mathrm{C}$, and $\Delta H=30.54 \mathrm{~kJ} / \mathrm{mol}$, that corresponds the melting of the NIA [21].

The DSC curve of AMB - NIA presents a broad exothermic signal between 185 and $198^{\circ} \mathrm{C}$, with a maximum at $188.5^{\circ} \mathrm{C}$ and $\Delta H=69.43 \mathrm{~kJ} / \mathrm{mol}$, corresponding to the melt with start of decomposition of the sample.

The thermal behaviour characteristics of AMB, NIA and AMB - NIA obtained by SDG procedure, have been investigated by thermogravimetry (TG) and are shown in Figs 6-8.

The TG curve for the pure AMB shows the $7.24 \%$ mass loss between $97-150^{\circ} \mathrm{C}$ corresponding to the evaporation of water. In the $184-208^{\circ} \mathrm{C}$ temperature range significant mass loss appeared representing $25.41 \%$ from mass of $\mathrm{AMB}$ due to the starting of decomposition. The next mass losses, 6.91 and 


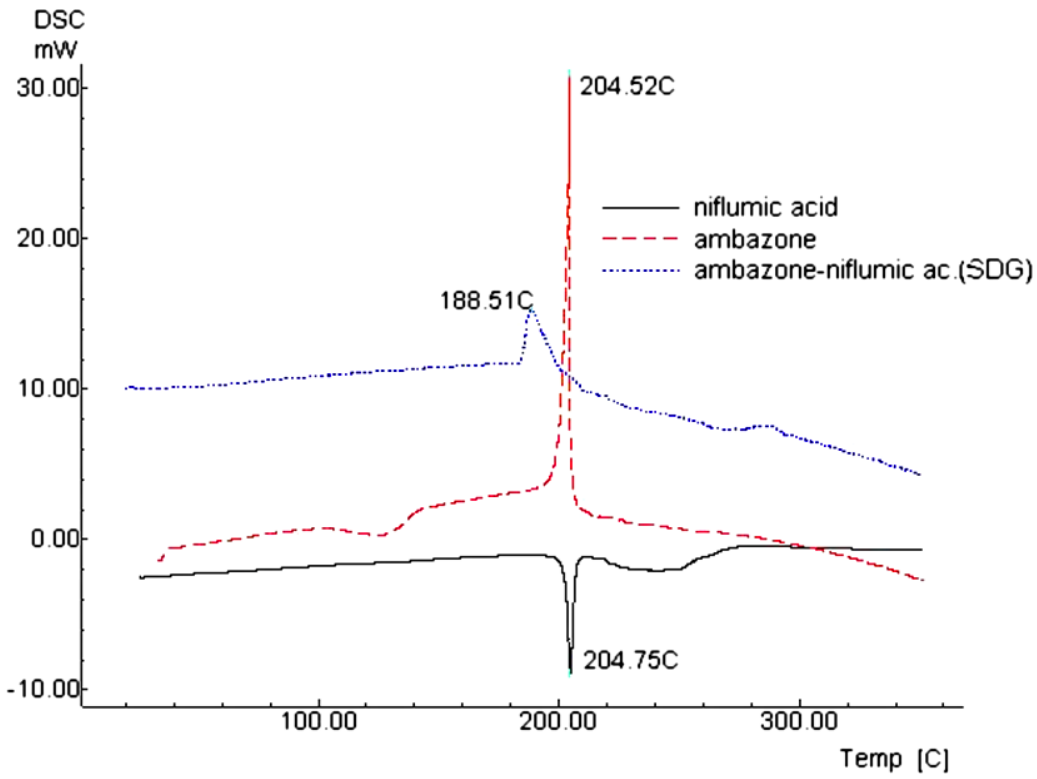

Fig. 5. DSC thermograms of pure AMB, NIA and AMB - NIA, obtained by SDG. (Colors are visible in the online version of the article; http://dx.doi.org/10.3233/SPE-2012-0570.)

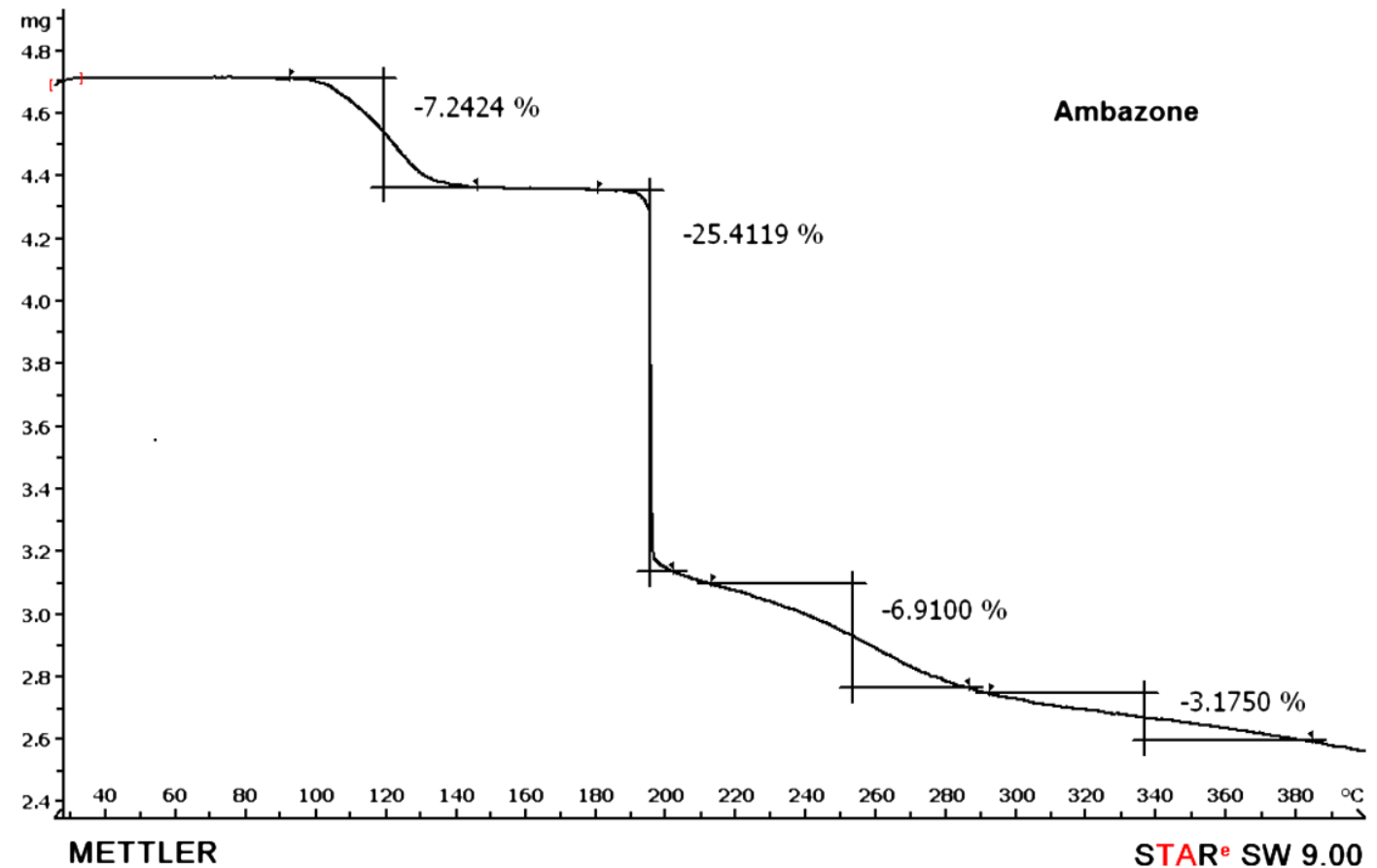

Fig. 6. The TG thermogram of AMB. (Colors are visible in the online version of the article; http://dx.doi.org/10.3233/SPE-20120570.) 


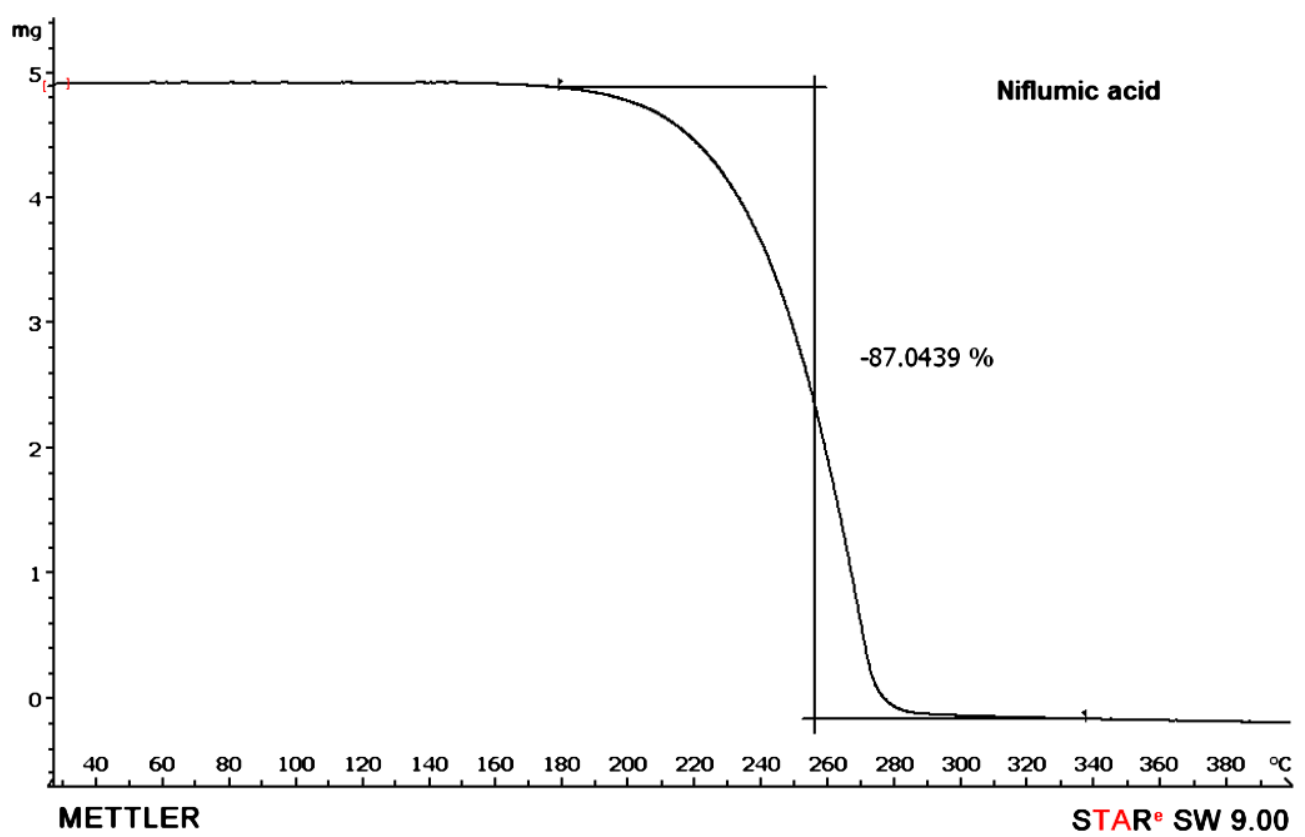

Fig. 7. The TG thermogram of NIA. (Colors are visible in the online version of the article; http://dx.doi.org/10.3233/SPE-20120570.)

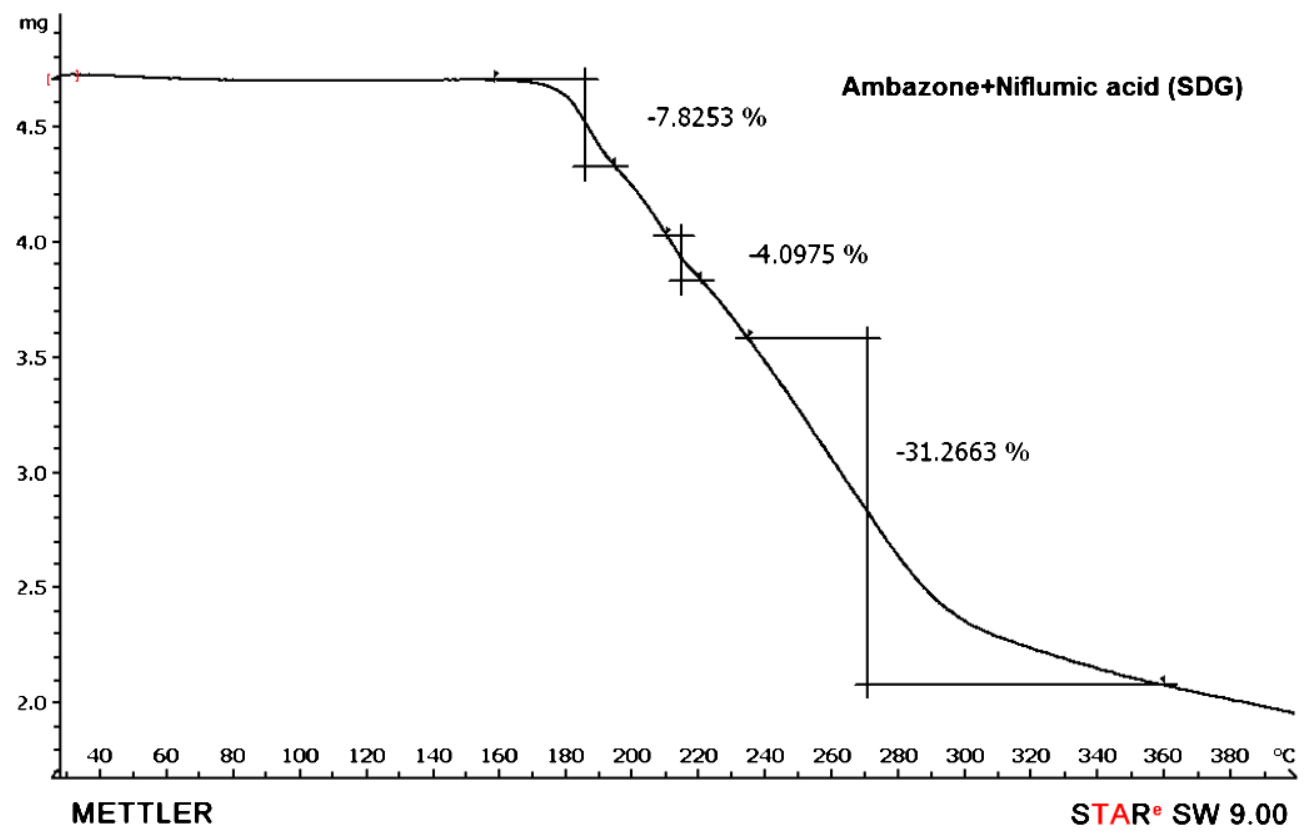

Fig. 8. TG thermogram of AMB - NIA, obtained by SDG. (Colors are visible in the online version of the article; http://dx.doi.org/ 10.3233/SPE-2012-0570.) 


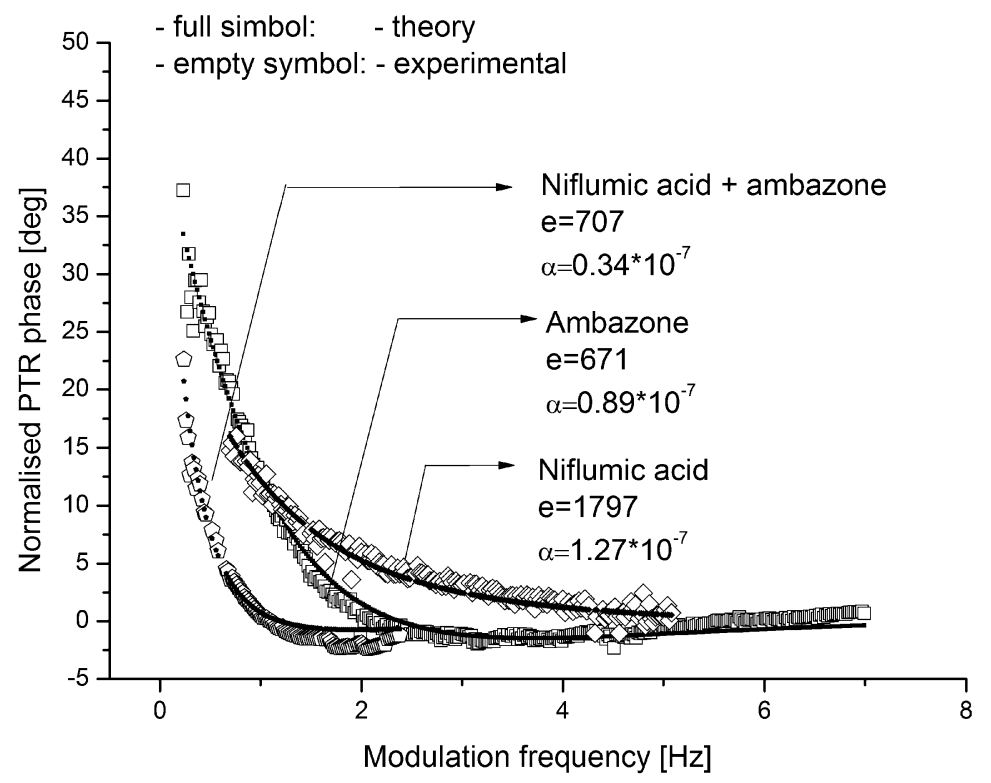

Fig. 9. Normalized PTR phase as a function of modulation frequency for AMB, NIA and AMB · NIA compounds.

$3.17 \%$ in the $216-288^{\circ} \mathrm{C}$ temperature range, respectively $294-386^{\circ} \mathrm{C}$ correspond to the elimination of the volatile components resulted from decomposition. The temperature ranges where mass losses occur are in agreement with DSC results.

The trace of thermogravimetric behaviour of the NIA shows an $87.04 \%$ mass loss in the $182-340^{\circ} \mathrm{C}$ temperature range, corresponding on the DSC curve to two endothermic peaks: a sharp one due to the melting, and a broader one due to decomposition of the NIA.

The thermogravimetric curve of the AMB - NIA shows the first mass loss, $7.82 \%$, in the range 168 $192^{\circ} \mathrm{C}$, followed by $4.09 \%$ mass loss in the range $213-224^{\circ} \mathrm{C}$. Last mass loss is the most significant, $31.26 \%$ and occurs in the $238-362^{\circ} \mathrm{C}$ temperature range.

The TG data are in good agreement with the DSC results and support the new obtained compound formation.

\subsection{Photothermal radiometry}

The PTR signal is a function of samples' thermal parameters and chopping frequency of radiation, see Fig. 9. The results (room temperature values of thermal diffusivity and effusivity) were obtained through a fitting procedure with the two mentioned thermal parameters as fitting parameters.

The PTR investigations leaded to values of dynamic thermal parameters out of the range of the values of these parameters for the starting materials. Such behavior can be ascribed to two phenomena: formation of a new compound and/or packing effects. Due to the fact that the pressure used to prepare the pellets was the same, we choose as more possible the first alternative. In fact, the formation of a new compound is also supported by the previous investigations.

\section{Conclusions}

- The FTIR spectra indicate the appearance of vibrations bands characteristic to $\mathrm{NH}_{2}{ }^{+}$and $\mathrm{NH}_{3}{ }^{+}$ groups corresponding to the protonation of the secondary and primary amine. Also new vibration 
bands appear. Consequently, a salt of ambazone and niflumic acid formation was confirmed.

- Thermal behaviour of pure substances and of AMB.NIA compounds are different, the distinct melting point and the decomposition mode certifies the new compound formation.

- New solid form that was prepared with SDG method combining the ambazone and niflumic acid. Based on X-ray powder diffraction the lattice parameters and the space group for the new compound were determined.

- The results obtained by photothermal radiometry can be considered also a support for the formation of a new compound.

\section{Acknowledgements}

The financial support of the PN 09-44 02 05/2009, PN II-ID-PCE-2011-0036 and POS CCE 536/2010 projects is greatly acknowledged.

\section{References}

[1] R. Amlacher, J. Baumgart, A. Hartl, H. Weber, H.J. Kuhnel, W. Schulze and H. Hoffmann, Influence of age on antileukemic action, subacute toxicity and tissue distribution of ambazone in B6D2F1 mice, Arch. Geschwulstforsch. 60(1) (1990), 11-18.

[2] R. Amlacher, A. Mackowiak, J. Baumgart, W. Schulze and H. Hoffmann, Influence of murine melanoma B16 on the distribution of ambazone in B6D2F1 mice, Pharmazie 45(5) (1990), 379-380.

[3] K. Balc, Y. Akkaya and S. Akyuz, An experimental and theoretical vibrational spectroscopic study on niflumic acid, a non-steroidal anti-inflammatory drug, Vibrational Spectroscopy 53 (2010), 239-247.

[4] J. Baumgart, N.V. Zhukovskaya and V.N. Anisimov, Carcinogenesis and aging. VIII. Effect of host age on tumour growth, metastatic potential, and chemotherapeutic sensitivity to 1.4-benzoquinoneguanyl hydrazonethiosemicarbazone (ambazone) and 5-fluorouracil in mice and rats, Exp. Pathol. 33(4) (1988), 239-248.

[5] H.G. Brittain, Polymorphism in Pharmaceutical Solids, Drugs and the Pharmaceutical Sciences, Vol. 192, 2nd edn, Informa Healthcare, New York, 2009.

[6] L. Cheng and M.C. Sanguinetti, Niflumic acid alters gating of HCN2 pacemaker channels by interaction with the outer region of S4 voltage sensing domains, Molecular Pharmacology 75(5) (2009), 1210-1221.

[7] A.P. Cleaves and E.K. Plyler, The infra-red absorption spectrum of methylamine vapor, J. Chem. Phys. 7 (1939), 563-569.

[8] M.C. Etter and D.A. Adsmond, The use of cocrystallization as a method of studying hydrogen bond preferences of 2-aminopyrimidine, J. Chem. Soc., Chem. Commun. 8 (1990), 589-591.

[9] M.C. Etter, S.M. Reutzel and C.G. Choo, Self-organization of adenine and thymine in the solid state, J. Am. Chem. Soc. 115 (1993), 4411-4412.

[10] I. Fichtner and W. Arnold, Antineoplastic effect of 1,4-benzoquinone guanylhydrazone thiosemicarbazone in experimental tumor models, Pharmazie 38(2) (1983), 130-131.

[11] I. Fulga, Ambazona: notiuni acceptate, informatii noi, posibile perspective, Pharmakon 56 (2006); available at: www. pharmakon.ro/inc/arhiva/2006_02/Ambazona.pdf.

[12] W. Gutsche, A. Hartl, J. Baumgart and W. Schulze, Antineoplastic activity and toxicity of dihydroambazone in comparison with ambazone (1,4-benzoquinone-guanyl hydrazone thiosemicarbazone), Pharmazie 45(1) (1990), 55-57.

[13] R.A. Heacock and L. Marion, The infrared spectra of secondary amines and their salts, Can. J. Chem. 34 (1956), 17821795.

[14] B.B. Koleva, T. Kolev, R.W. Seidel, M. Spiteller, H. Mayer-Figge and W.S. Sheldrick, Self-assembly of hydrogensquarates: crystal structures and properties, J. Phys. Chem. A 113(13) (2009), 3088-3095.

[15] H.J. Kuhnel, R. Amlacher, J. Baumgart and W. Schulze, Distribution of ${ }^{14}$ C-ambazone in normal and leukemia P388bearing mice, Arch. Geschwulstforsch. 58(4) (1988), 217-222.

[16] H.J. Kuhnel, R. Amlacher, K. Kramarczyk and W. Schulze, Pharmacokinetics of ${ }^{14}$ C-ambazone in rats, Pharmazie 43(3) (1988), 197-199.

[17] A.R. Ling and J.L. Baker, Halogen derivatives of quinone. Part III. Derivatives of quinhydrone, J. Chem. Soc. 63 (1893), 1314-1327. 
[18] G. Lober, K. Geller, H. Hanschmann, L.M. Popa, R. Repanovici, R. Iliescu, W. Romer, B. Janke and V. Kleinwachter, Cationic anticancer drugs and their modes of action, Physiologie 26(4) (1989), 305-316.

[19] K. Nakanishi, Infrared Absorption Spectroscopy - Practical, Holden-Day, San Francisco, CA, 1962.

[20] M. Otsuka, K. Otsuka and N. Kaneniwa, Relation between polymorphic transformation pathway during grinding and the physicochemical properties of bulk powders for pharmaceutical preparations, Drug Dev. Ind. Pharm. 20 (1994), 16491660.

[21] J.J. Pinvidic, A. Gonthier-Vassal, H. Szwarc, R. Ceolin, P. Toffoli, J.M. Teulon and C. Guechot, Niflumic acidmorniflumate phase diagram: I. Study of the components, Thermochimica Acta 153(1) (1989), 37-45.

[22] J. Pirttimaki, E. Laine, J. Ketolainen and P. Paronen, Effects of grinding and compression on crystal structure of anhydrous caffeine, Int. J. Pharm. 95 (1993), 93-99.

[23] N. Shan, F. Toda and W. Jones, Mechanochemistry and co-crystal formation: effect of solvent on reaction kinetics, Chem. Commun. (Camb.) 20 (2002), 2372-2373.

[24] G. Socrates, Infrared and Raman Characteristic Group Frequencies: Tables and Charts, 3rd edn, Wiley, West Sussex, 2001.

[25] G.H. Tilve, J.K. Lengade, A.V. Bavadekar and K.G. Nair, Niflumic acid in the management of rheumatoid and osteoarthritis, J. Postgraduate Medicine 22(3) (1976), 124-129. 


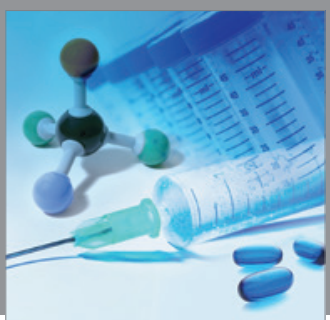

International Journal of

Medicinal Chemistry

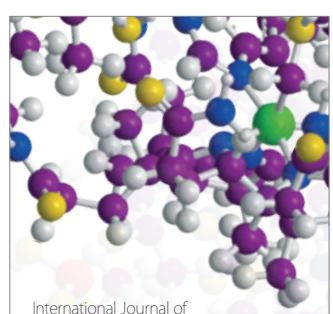

Carbohydrate Chemistry

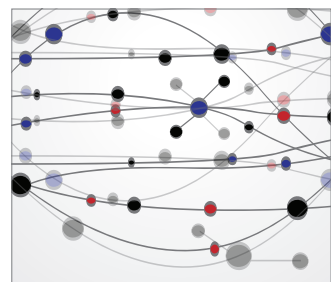

The Scientific World Journal
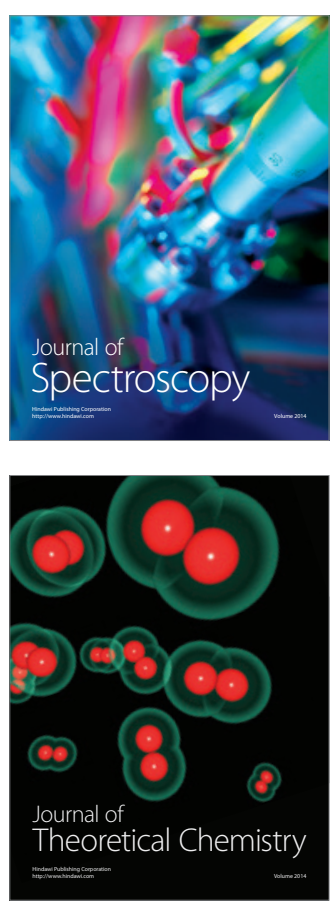
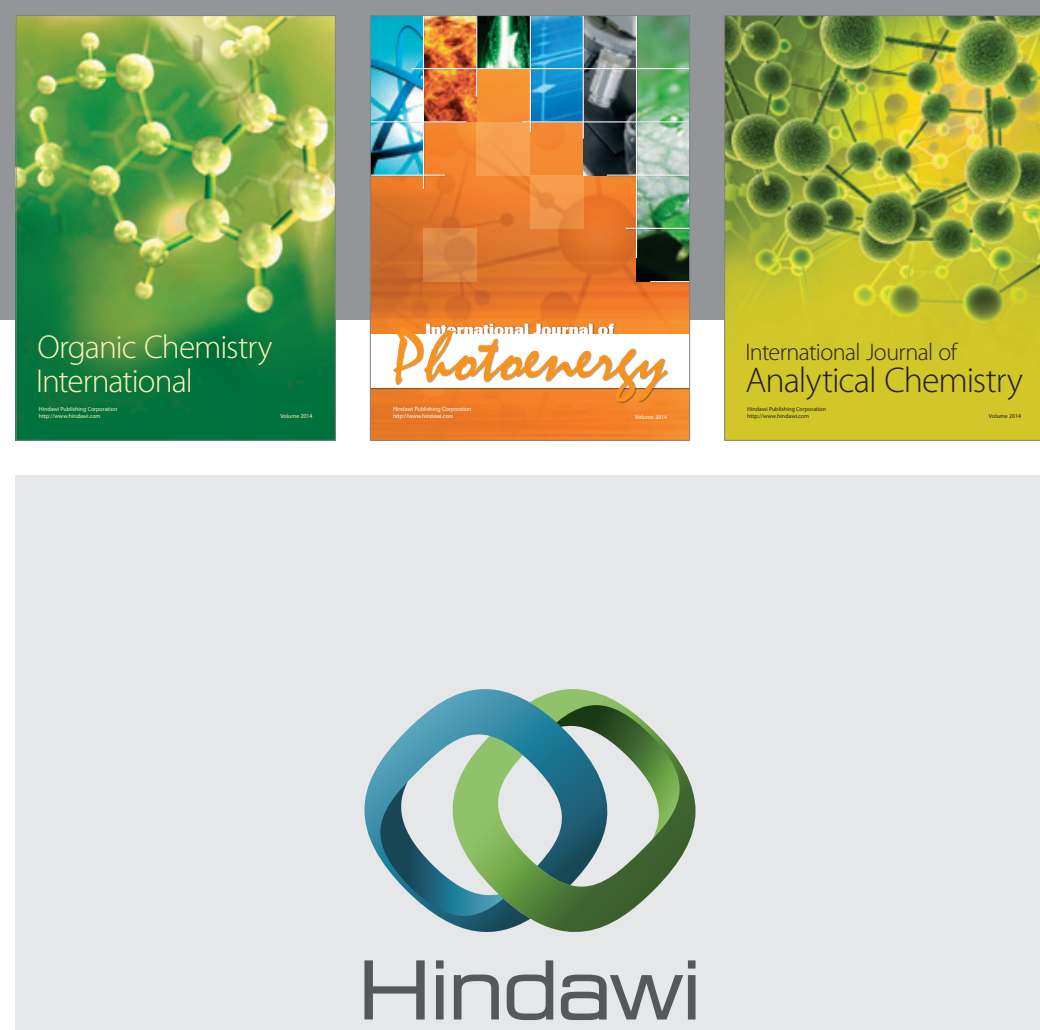

Submit your manuscripts at

http://www.hindawi.com
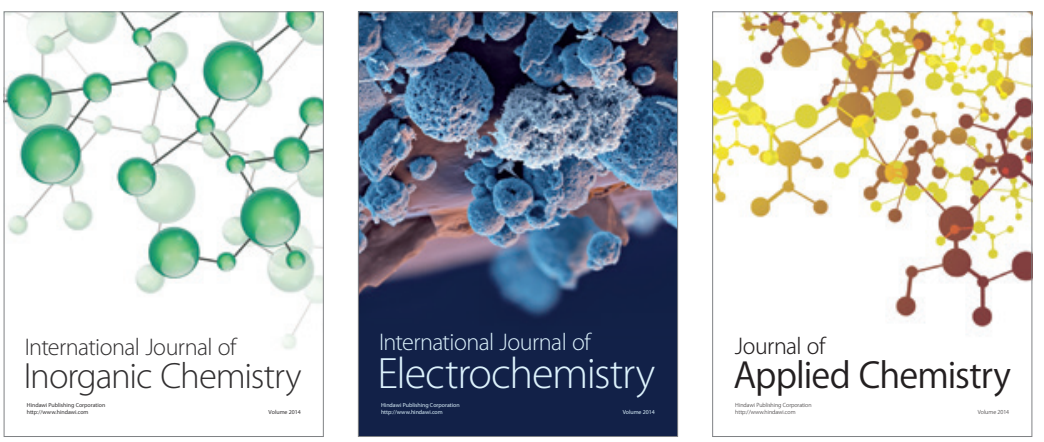

Journal of

Applied Chemistry
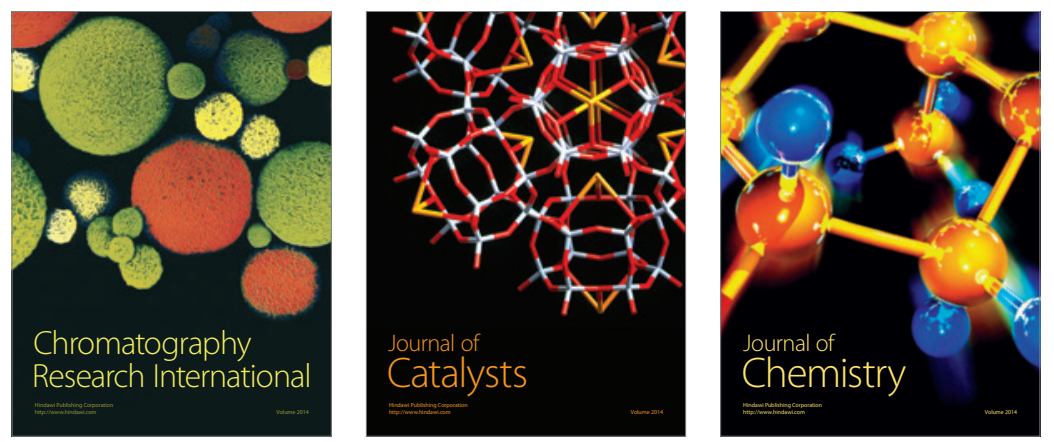
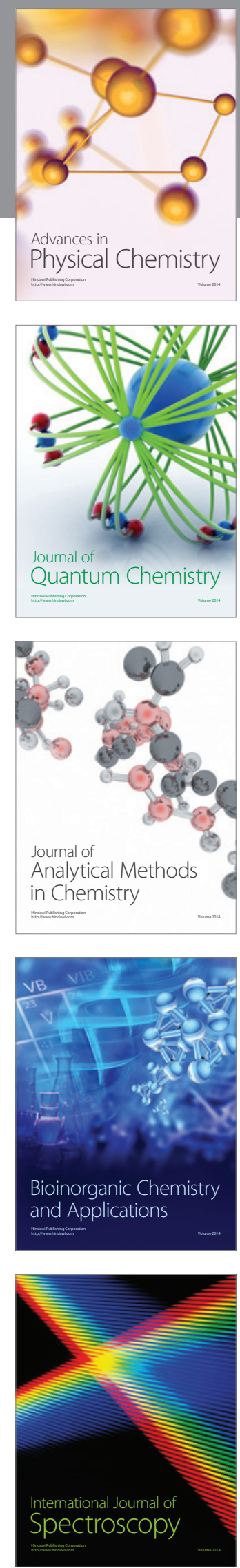